\title{
Assessment of free-hand transperineal targeted prostate biopsy using multiparametric magnetic resonance imaging-transrectal ultrasound fusion in Chinese men with prior negative biopsy and elevated prostate-specific antigen
}

Huibo Lian ${ }^{1,4+}$, Junlong Zhuang ${ }^{1,4+}$, Wei Wang ${ }^{1,4^{*}}$, Bing Zhang ${ }^{2}$, Jiong Shi ${ }^{3}$, Danyan Li ${ }^{2}$, Yao Fu ${ }^{3}$, Xuping Jiang ${ }^{5}$, Weimin Zhou ${ }^{5}$ and Hongqian Guo ${ }^{1,4^{*}}$

\begin{abstract}
Background: To evaluate the role of free-hand transperineal targeted prostate biopsy using multiparametric magnetic resonance imaging-transrectal ultrasound (mpMRI-TRUS) fusion in Chinese men with repeated biopsy.

Methods: A total of 101 consecutive patients suspicious of prostate cancer (PCa) at the mpMRI scan and with prior negative biopsy and elevated PSA values were prospectively recruited at two urological centers. Suspicious areas on mpMRI were defined and graded using PI-RADS score. Targeted biopsies (TB) were performed for each suspicious lesion and followed a 12-core systematic biopsy (SB). Results of biopsy pathology and whole-gland pathology at prostatectomy were analyzed and compared between TB and SB. The risk for biopsy positivity was assessed by univariate and multivariate logistic regression analysis.
\end{abstract}

Results: Fusion biopsy revealed PCa in 41 of 101 men (40.6\%) and 25 (24.8\%) were clinically significant. There was exact agreement between TB and SB in 74 (73.3\%) men. TB diagnosed 36\% more significant cancer than SB (22 vs 13 cases, $P=0.012)$. When TB were combined with SB, an additional 14 cases (34.1\%) of mostly significant PCa (71.4\%) were diagnosed $(P=0.036)$. TB had greater sensitivity and accuracy for significant cancer than SB in 26 men with whole-gland pathology after prostatectomy. PI-RADS score on mpMRI was the most powerful predictor of PCa and significant cancer.

Conclusions: Free-hand transperineal TB guided with MRI-TRUS fusion imaging improves detection of clinical significant PCa in Chinese men with previously negative biopsy. PI-RADS score is a reliable predictor of PCa and significant cancer.

Keywords: Magnetic resonance imaging, Prostate cancer, Repeat biopsy, Targeted biopsy, Transrectal ultrasound

\footnotetext{
*Correspondence: wawe9999@163.com; dr.guohongqian@gmail.com

${ }^{\dagger}$ Equal contributors

'Department of Urology, Drum Tower Hospital, Medical School of Nanjing

University, 321 Zhongshan Road, Nanjing 210008, Jiangsu, People's Republic

of China

Full list of author information is available at the end of the article
} 


\section{Background}

Since the 1980s, transrectal ultrasound (TRUS) guided systematic prostate biopsy is performed on patients with abnormal serum prostate-specific antigen (PSA) or suspicious digital rectal examination [1]. This conventional method has been shown to have limited sensitivity for detecting prostate cancer ( $\mathrm{PCa})$, which of the falsenegative rate may be as high as $47 \%$ [2]. PSA related anxiety and repeated biopsy dilemma consist in many of the men with negative biopsies and persistently elevated serum PSA levels [3]. Approximately 38\% of them undergo a repeat biopsy within 5 years with cancer detection only in an additional 13 to $41 \%$ [4].

In order to improve biopsy sensitivity, the concept of targeted biopsy (TB) on suspicious areas through magnetic resonance imaging (MRI) guidance was established [5]. Although multiparametric MRI (mpMRI) offered an increased sensitivity and specificity on prostate biopsy guidance, the disadvantage of time-consuming and equipment-specialization made it not widely used [6]. Recently, MRI-TRUS fusion technique has been developed and proposed, because of its combination of the soft tissue resolution of MRI and the practicability of TRUS [7]. The mpMRI-TRUS image fusion biopsy system is a novel fusion technology which not only provides visualization of both recorded multiplanar reconstruction images on that one monitor, but also real-timely makes diagnostic or procedural decisions [8]. Using one such fusion device, we got initial encouraging result for targeted prostate biopsy as reported by other researchers [9-11]. Unfortunately, targeted MRI-TRUS fusion biopsy has not been well evaluated with free-hand transperineal approach, especially in Asian men with previously negative biopsy.

We present double center results to evaluate the impact of using mpMRI-TRUS image fusion technology for free-hand transperineal TB in Chinese men with prior negative biopsy and elevated PSA, and compare biopsy performance between TB and 12-core systematic biopsy $(\mathrm{SB})$ in the cancer detection.

\section{Methods}

\section{Study population}

After the approval of institutional review board, a prospective study of free-hand transperineal TB guided with MRI-TRUS fusion imaging was performed at two Chinese urological centers from May 2014 to March 2016 (Drum Tower Hospital, Medical School of Nanjing University, and the Affiliated Yixing people's Hospital of Jiangsu University). One hundred and one consecutive patients with at least one prior negative prostate biopsy and persistently elevated serum PSA levels were included. All of them were evaluated with prostate mpMRI and considered having at least one suspicious area in mpMRI images.

\section{Multiparametric MRI}

All mpMRI was performed using a 3 Tesla MRI scanner (Achieva; Philips Medical System, The Netherlands) with a 32-channel phased array coil. The protocol of acquisition of different MRI sequences was recently published [12]. Images analyses were performed and supervised by two experienced uroradiologists. Suspicious areas were defined and a likelihood score from 2 to 5 for each lesion was provided according to the Prostate Imaging Reporting and Data System (RI-PADS) [13] based on the European Society of Urogenital Radiology prostate MRI guidelines [14]. We didn't use the PI-PADS Version 2 [15], because the new system had not been well validated and most of the patients' lesions were evaluated before its publication.

\section{Biopsy procedure}

All biopsies were performed with an mpMRI-TRUS fusion guided biopsy technique (RVS ${ }^{\oplus}$, Real-time Virtual Sonography, Hitachi Medical Corporation, Tokyo, Japan) and 18-G automatic biopsy guns with $22 \mathrm{~mm}$ specimen size (Bard Magnum; Bard Medical, Covington, GA, USA) as described previously [12]. In brief, morphological MRI data was loaded into the biopsy system and suspicious areas were marked on high resolution transversal T2 W sequences (Fig. 1 a-d). Then, ultrasound probe with magnetic position sensor was used to get the TRUS images. During fusing two kinds of images, the MR images reconstructed from the MRI volume data were corresponded to the ultrasound sagittal images using internal urethral orifice as the fiducial landmark. Thus, mpMRI data with the marked suspicious lesions were real-timely superimposed on the TRUS images at the same monitor.

Then, the biopsy started with TB using the free-hand transperineal technique without the guide of template by one experienced urologist. Cancer-suspicious lesions identified on MRI were semiautomatically displayed on the real-time TRUS image to guide biopsy needle [10]. During free-hand transperineal biopsy procedure, the puncture point was chose to keep away from pubis and adjacent organs, and make the needle correspond to the ultrasound sagittal images (Fig. 1 e). Every targeted lesion was biopsied at least each one core in axial and sagittal planes. Standard 12-core SB using same transperineal approach was carried out afterwards by another experienced urologist who was blinded to the MRI targeted lesions. During the biopsy procedure, all patients with lithotomy position underwent general anesthesia using a larynx mask.

\section{Pathological analysis}

All biopsies and prostatectomy whole mount pathology were examined and analyzed by two senior pathologists 


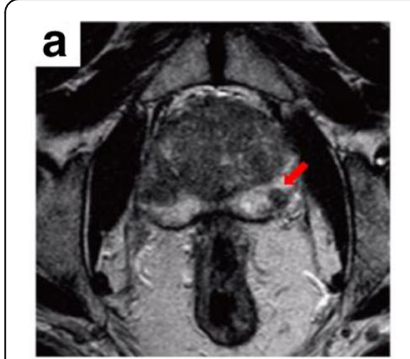

T2WI

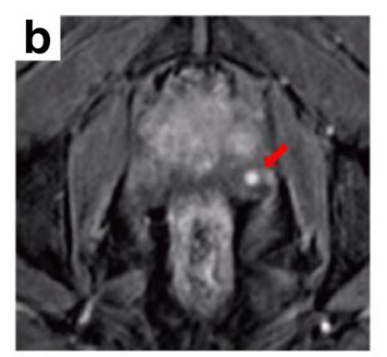

DCE

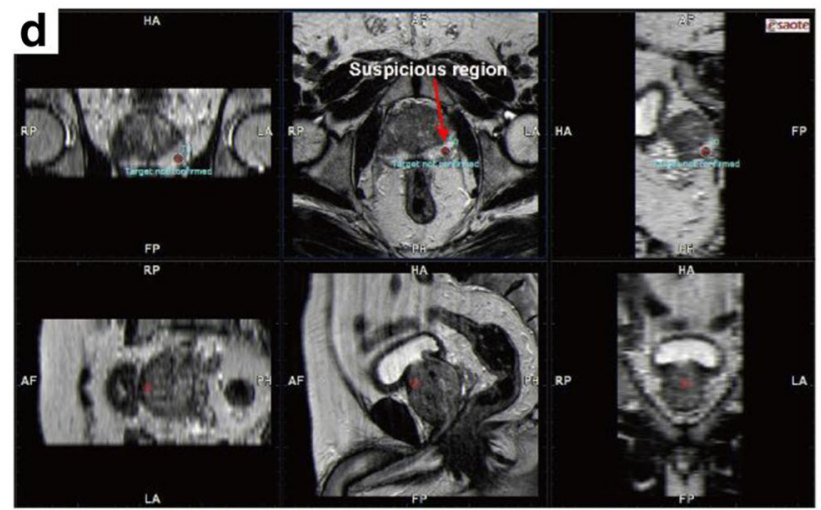

Suspicious region marked

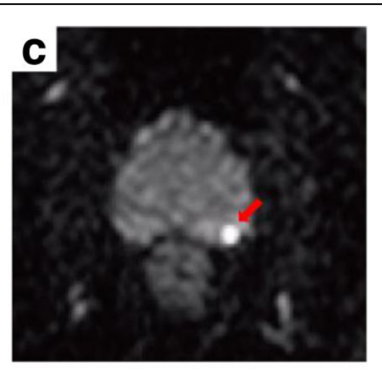

DWI

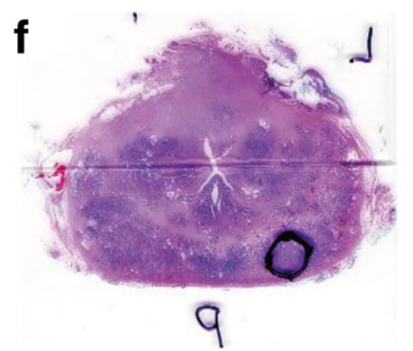

Whole-gland

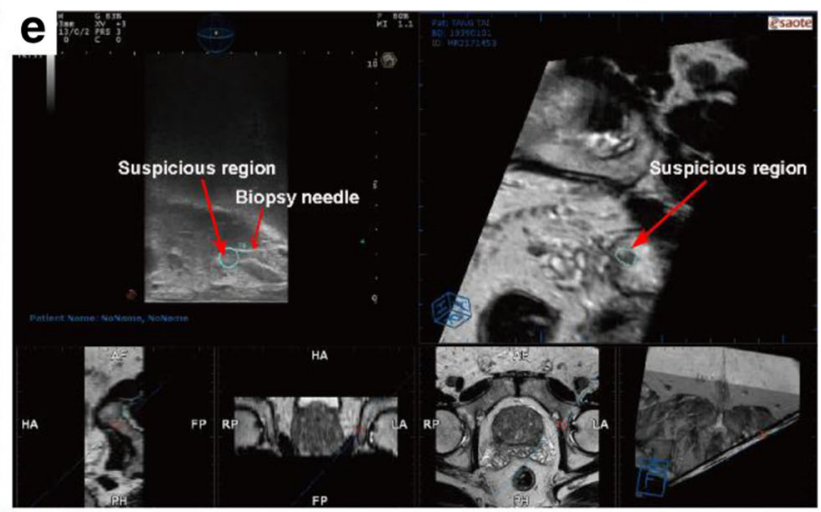

Targeted biopsy

Fig. 1 Steps for mpMRI and TRUS fusion guided targeted prostate biopsy and whole-gland prostatectomy pathology in a typical patient with negative biopsy two years ago but elevated prostate-specific antigen. A-C, The lesion (red arrow) was detected in different mpMRI sequences and was scored as "probably malignant" (score 4 of 5). D-E, mpMRI data was loaded into the biopsy system and suspicious region (greeb circle) was marked on high resolution transversal T2 W sequences before biopsy. Then, the targeted biopsy started using the free-hand transperineal technique guided by mpMRI and TRUS fusion images (red arrow). F, Targeted cores revealed Gleason $3+4$ tumor in the lesion ( $90 \%$ core involvement).

Whole-gland pathology was performed after prostatectomy and the index lesion (black circle) had the same pathology result with targeted biopsy

(J.S. and Y.F.). The highest Gleason score from the TB or the standard 12-core SB was determined for each patient. Clinically significant cancer on biopsy was defined as Gleason score $3+4$ or higher or Gleason score 6 with maximal cancer core length $\geq 4 \mathrm{~mm}[16,17]$. This definition was selected in an effort to incorporate both grade and volume, and avoid the bias caused by multiple cores from the same tumor.

Each prostatectomy specimen was processed using the modified Stanford technique, with $5 \mathrm{~mm}$ transverse step-sectioned samples taken from the apex to the base and the sagittal section of the distal 5-8 $\mathrm{mm}$ of the apex and base [18]. The step sectioned specimens were denoted as the apex, middle, or base of the prostate for analyses of three equal trisections of the prostate. Pathologists were blinded to the MRI and TRUS imaging results. The index tumor lesion in prostatectomy specimens was defined as the lesion with extraprostatic extension, the highest Gleason score, or the largest volume if Gleason scores were the same, in order of priority. The pathology slide with the greatest cross-section of the index lesion was used for location matching analysis. The tumor center of the index lesion was defined as the point of intersection of the lesion height and width dimensions and was registered retrospectively in the 27-ROI schema by urologists [19]. Significant PCa at prostatectomy histology was defined using active surveillance criteria (total tumor volume $\geq 0.7 \mathrm{ml}$ or Gleason score $>3+4$ ) [20]. The Steps for mpMRI and TRUS fusion guided targeted prostate biopsy and whole-gland prostatectomy pathology are outlined in Fig. 1.

\section{Data statistics}

Statistical analyses were performed using SPSS version 17.0 (SPSS, Inc., Chicago, IL). The Fisher's exact test was used to compare categorical variables. Univariate analysis was applied with one-way ANOVA test. Multivariate logistic regression analysis was performed to identify potential predicted factors for the positive result of biopsy. Data was presented as mean \pm SD. A $P$ value $<0.05$ was considered statistically significant.

\section{Results}

Patient demographics and summary of fusion-guided biopsy findings are shown in Table 1. A total of 101 patients with prior negative biopsy and elevated PSA 
Table 1 Patient demographics and summary of fusion-guided biopsy findings

\begin{tabular}{|c|c|c|c|}
\hline & All patients & Men with PCa & Prostatectomy cohort \\
\hline Men, no. & 101 & $41(40.6)$ & $26(25.7)$ \\
\hline Age, year & $68.9 \pm 8.1$ & $67.8 \pm 8.0$ & $65.2 \pm 7.2$ \\
\hline $\mathrm{PSA}, \mathrm{ng} / \mathrm{ml}$ & $10.8 \pm 6.1$ & $11.3 \pm 6.3$ & $11.2 \pm 6.3$ \\
\hline Prostate volume, $\mathrm{ml}$ & $42.1 \pm 15.3$ & $39.4 \pm 13.6$ & $35.1 \pm 11.8$ \\
\hline Prior negative biopsy, no. & $1.5 \pm 0.7$ & $1.4 \pm 0.6$ & $1.4 \pm 0.6$ \\
\hline MRI lesions per patient, no. & $1.9 \pm 1.0$ & $2.1 \pm 1.1$ & $2.4 \pm 1.0$ \\
\hline \multicolumn{4}{|l|}{ PI-RAD score, no. (\%) } \\
\hline 2 & $13(12.9)$ & $1(1.0)$ & $0(0)$ \\
\hline 3 & $31(30.7)$ & $3(3.0)$ & $0(0)$ \\
\hline 4 & $36(35.6)$ & $19(18.8)$ & $12(11.9)$ \\
\hline 5 & $21(20.8)$ & $18(17.8)$ & $14(13.9)$ \\
\hline TB cores per patiens & $4.2 \pm 1.5$ & $4.8 \pm 1.6$ & $4.9 \pm 1.8$ \\
\hline Insignificant PCa & - & $16(15.8)$ & $6(5.9)$ \\
\hline Significant PCa & - & $25(24.8)$ & $20(19.8)$ \\
\hline \multicolumn{4}{|l|}{ Gleason score, no. (\%) } \\
\hline Gleason 6 & - & $17(16.8)$ & $6(5.9)$ \\
\hline Gleason $7(3+4)$ & - & $9(8.9)$ & $8(7.9)$ \\
\hline Gleason $7(4+3)$ & - & $7(6.9)$ & $5(5.0)$ \\
\hline Gleason $\geq 8$ & - & $8(7.9)$ & $7(6.9)$ \\
\hline
\end{tabular}

Continuous variables reported as mean \pm standard deviation

PSA prostate-specific antigen, $M R I$ magnetic resonance imaging, $P C a$ prostate cancer, $S B$ systematic biopsy, TB targeted biopsy

were suspected to have PCa with a PI-RADS score between 2 and 5 according to mpMRI examination. The mean age of the patient population was 68.9 years (SD 8.1) and mean number of MRI lesions was 1.9 (SD 1.0). The mean pre-fusion-guided biopsy PSA level was $10.8 \mathrm{ng} / \mathrm{ml} \mathrm{(SD} \mathrm{6.1)} \mathrm{and} \mathrm{prostate} \mathrm{volume} \mathrm{was} 42.1 \mathrm{ml}$ (SD 15.3). The mean number of targeted biopsy cores per patient was 4.2 (SD 1.5). Of 101 suspected patients, 41 (40.6\%) were diagnosed PCa, including 16 (15.8\%) insignificant and $25(24.8 \%)$ significant cancers. Twentysix patients who ultimately underwent prostatectomy were analyzed as a subgroup. Compared with all biopsy populaiton, patients who underwent prostatectomy were younger (65.2 vs 68.9 years, $P=0.028)$, had smaller prostate volumes ( 35.1 vs $42.1 \mathrm{ml}, P=0.015$ ), had more MRI lesions (2.4 vs $1.9, P=0.029)$, and had more TB cores (4.9 vs $4.2, P=0.040$ ).

The comparative pathologic outcomes of prostate systemic biopsy and targeted biopsy are shown in Table 2 and Additional file 1: Table S1. Seventy-four patients $(60+4+10)$ of the total population $(73.3 \%)$ demonstrated exact agreement between $\mathrm{TB}$ and SB. TB diagnosed a similar $\mathrm{PCa}$ number (31 cases) to $\mathrm{SB}$ (27 cases). However, TB diagnosed 36\% more significant cancers than SB ( 22 vs 13 cases, $P=0.012)$. Among the 16 cases $(4+10+2,15.8 \%)$ in which TB revealed a higher risk category from SB group, 12
$(10+2,75 \%)$ were upgraded to significant cancers; whereas in 11 cases $(8+2+1,10.9 \%)$ which SB demonstrated a higher risk category from TB group, only $3(2+1,27.3 \%)$ were upgraded to significant cancers $(P=0.022)$. In addition, the utility of TB alone lead to 10 less cases of cancer (24.4\%), only 2 $(20 \%)$ of these were significant. However, SB alone missed 14 cases of cancer (34.1\%) and 10 (71.4\%) were significant $(P=0.036)$. In other words, when TB were combined with $\mathrm{SB}$, an additional 14 cases of mostly significant PCa (10 cases) were diagnosed.

The subgroup of 26 patients who underwent prostatectomy was also analyzed because pathology results

Table 2 Comparison of pathology from systematic biopsy and targeted biopsy for prostate cancer

\begin{tabular}{|c|c|c|c|c|}
\hline & \multicolumn{3}{|l|}{ SB } & \multirow[b]{2}{*}{ Totals } \\
\hline & $\begin{array}{l}\text { No } \\
\text { cancer }\end{array}$ & $\begin{array}{l}\text { Insignificant } \\
\text { cancer }\end{array}$ & $\begin{array}{l}\text { Significant } \\
\text { cancer }\end{array}$ & \\
\hline \multicolumn{5}{|l|}{ TB } \\
\hline No cancer. & 60 & 8 & 2 & 70 \\
\hline $\begin{array}{l}\text { Insignificant } \\
\text { cancer }\end{array}$ & 4 & 4 & 1 & 9 \\
\hline $\begin{array}{l}\text { Significant } \\
\text { cancer }\end{array}$ & 10 & 2 & 10 & 22 \\
\hline Totals & 74 & 14 & 13 & 101 \\
\hline
\end{tabular}

SB systematic biopsy, TB targeted biopsy 
from TB and SB could be compared against the wholegland prostatectomy pathology (Table 3 and Additional file 1: Table S1). Within this subcohort, nine patients (the sum of all "no cancer" values for SB, $1+1+1+6$ ) were diagnosed with $\mathrm{PCa}$ preoperatively only by $\mathrm{TB}$, of whom 6 (66.7\%) were significant cancer on whole-gland pathology. By contrast, 4 patients were diagnosed with PCa only by SB, only 1 (25\%) were significant cancer on whole-gland pathology. When assessing the ability of preoperative biopsy to predict whole-gland pathology significance, the sensitivity of TB were $85 \%$ versus $45 \%$ for SB $(P=0.019)$, while the specificities were same (83.3\%). The total accuracy of TB were $84.6 \%$ versus $53.8 \%$ for SB $(P=0.034)$.

In order to identify any potential predictor associated with detection of $\mathrm{PCa}$ and significant cancer, univariate and multivariate analysis were performed (Table 4). PIRADS score was significantly correlated with both the $\mathrm{PCa}$ and significant $\mathrm{PCa}$ (both $P<0.001$ ). Age, MRI lesions and PSA value of patients was only correlated with $\mathrm{PCa}(P=0.028, P<0.001$ and $P=0.03)$. The further multivariate analysis revealed that PSA value and PIRADS score were independent predictive factors of the positive biopsy of $\mathrm{PCa}(P=0.004, \mathrm{OR}=1.22 ; P=0.001$, $\mathrm{OR}=3.64)$. Moreover, patients with high PI-RADS scores $(4,5)$ had an over 10 -fold higher risk of positive biopsy compared to those with low PI-RADS scores $(2,3)$. Additional file 2: Figure S1 also showed a strong relationship between PI-RADS score and biopsy results.

\section{Discussion}

Imaging techniques, mainly mpMRI, have developed as an accurate modality in PCa detection. Lesions identified

Table 3 Comparison of whole-mount prostatectomy outcome with target biopsy and systematic biopsy pathology for prostate cancer

\begin{tabular}{llllll}
\hline & \multicolumn{3}{l}{ Whole-Mount Pathology (Prostatectomy) } & \\
\cline { 2 - 4 } & Insig cancer & Sig cancer & Totals \\
\hline TB & SB & SB & & \\
& No cancer & 0 & No cancer & 0 & 4 \\
& Insig cancer & 2 & Insig cancer & 1 & \\
& Sig cancer & 1 & Sig cancer & 0 & \\
Insig cancer & No cancer & 1 & No cancer & 1 & 4 \\
& Insig cancer & 1 & Insig cancer & 1 & \\
& Sig cancer & 0 & Sig cancer & 0 & \\
Sig cancer & No cancer & 1 & No cancer & 6 & 18 \\
& Insig cancer & 0 & Insig cancer & 2 & \\
Totals & Sig cancer & 0 & Sig cancer & 9 & \\
\hline SB systematc & 6 & & 20 & & 26 \\
\hline
\end{tabular}

SB systematic biopsy, TB targeted biopsy, Insig Insignificant, Sig significant
Table 4 Univariate and multivariate analysis (logistic regression) predicting prostate cancer and clinically significant cancer

\begin{tabular}{|c|c|c|c|c|}
\hline & \multicolumn{3}{|c|}{ Diagnosed with PCa } & \multirow{3}{*}{$\begin{array}{l}\text { Sig PCa } \\
\text { Univariate } \\
P \text { value }\end{array}$} \\
\hline & \multirow{2}{*}{$\begin{array}{l}\text { Univariate } \\
P \text { value }\end{array}$} & \multicolumn{2}{|c|}{ Multivariate } & \\
\hline & & $P$ value & OR (95\% Cl) & \\
\hline Age & 0.028 & 0.847 & $1.01(0.93-1.10)$ & 0.812 \\
\hline PSA & $<0.001$ & 0.004 & $1.22(1.07-1.39)$ & 0.070 \\
\hline Prostate volume & 0.114 & - & - & 0.397 \\
\hline Prior negative biopsy & 0.541 & - & - & 0.483 \\
\hline MRI lesions per patient & 0.030 & 0.193 & $1.67(0.77-3.59)$ & 0.953 \\
\hline Biopsy cores & 0.165 & - & - & 0.587 \\
\hline PI-RAD score & $<0.001$ & 0.001 & $3.64(1.74-7.63)$ & $<0.001$ \\
\hline $4+5$ vs $2+3$ & $<0.001$ & $<0.001$ & $10.94(3.0-40.1)$ & $<0.001$ \\
\hline
\end{tabular}

$P C a$ prostate cancer, Sig significant, PSA prostate-specific antigen,

$M R I$ magnetic resonance imaging

on mpMRI correlate with tumor location on radical prostatectomy specimens [21]. Real-time fusion of mpMRI and TRUS images of the prostate is feasible and potentially able to identify cancerous regions for subsequent biopsy. This kind of biopsy can be performed using MRI localization information without requiring the cost, difficulties, or inconvenience of an MRI suite or MRI-compatible equipment. This double center prospective study evaluated the impact of real time freehand transperineal targeted prostate biopsy guided by MRI-TRUS fusion imaging and made comparisons of biopsy performance between TB and traditional 12-core SB in Chinese men with prior negative biopsy sessions.

Our study indicated that $\mathrm{PCa}$ detection rate of $\mathrm{TB}$ and SB was 30.7 and $26.7 \%$ respectively, while the overall rate increased to $40.6 \%$ when combined the two approaches. With a mean of only 16.2 biopsies, we achieved a comparable overall detection rate to the others. Taira et al. reported a cancer yield ranging from 34.4 to $55.5 \%$ for men with 1,2 , and $\geq 3$ prior negative biopsies [2]. They used transperineal template guided mapping biopsy approach with an average of 54 cores. Walz et al. showed a cancer detection rate of $41 \%$ by using a 24-core transrectal saturation biopsy in men with at least two prior negative 8-core biopsies [4]. It indicated that MRI-TRUS fusion guided free-hand transperineal biopsy with lower cores obtained higher or almost cancer detection rate compared to transperineal template mapping biopsy or transrectal saturation approach. Our result was similar to Brock's, with a TB detection rate of $26.7 \%$ and overall rate of $40.6 \%$ by using transrectal $\mathrm{MRI} /$ real-time elastography fusion biopsy [22]. Besides, our overall cancer detection rate seemed higher than Sonn's result of 34\% [23], who used transrectal MRI-TRUS fusion biopsy in men with one or more previously negative biopsies and elevated PSA levels. We considered that the inconsistent result was 
because of the different biopsy pathway and patient demography, with older mean age and higher average PSA level in our cohort.

Current evidence demonstrates the improved sensitivity for detecting high grade or clinically significant $\mathrm{PCa}$ using MRI-TRUS fusion guided TB than with 12-core SB $[9,10,24]$. In this study, TB significantly increased the detection of significant PCa while decreasing the detection of insignificant cancer compared with SB in a repeat biopsy setting. When using the whole-gland pathology significance as the "gold standard", TB had a greater accuracy than SB for significant cancer on prostatectomy and a higher sensitivity of $85 \%$ versus $45 \%$. Thus, our results demonstrated that TB could significantly change the distribution of clinical significance in repeated biopsy patients diagnosed with $\mathrm{PCa}$ toward diagnosis of more significant disease.

The European Society of Urogenital Radiology (ESUR) published the PI-RADS to standardize the MRI scoring system in 2012 [14], which had been validated in primary and repeat biopsy cohorts [7, 25]. Portalez, D et al. considered that ESUR scoring system provided a clinically relevant stratification of the risk of showing $\mathrm{PCa}$ in the challenging situation of repeat biopsies [25]. Brock $\mathrm{M}$ et al. reported that the prediction of $\mathrm{PCa}$ and significant cancer was calculated with an AUC of 0.79 and 0.81 for PI-RADS score in lesion of repeat biopsies. Sonn GA et al. showed that image grade [26] of suspicion on MRI was the most powerful predictor of significant cancer on multivariate analysis [23]. In our cohort, using univariate and multivariate analysis, PI-RADS score was proven to be the strongest predictor of PCa or significant cancer as well (Table 4). Moreover, a strong relationship existed between PI-RADS score and biopsy results (Additional file 2: Figure S1). Patients with high PI-RADS scores $(4,5)$ had an over 10-fold higher risk of biopsy positivity compared to those with low PI-RADS scores $(2,3)$.

It is well known that the incidence of infectious complications following TRUS guided transrectal prostate biopsy is steadily increased. In a European randomized trial of 10,474 prostate needle biopsies, the febrile complication rate was as high as $4.2 \%$ [27]. In the patients of repeated transrectal biopsies, there was a higher chance of acquiring sepsis with organisms resistant to standard antibiotics, such as multiresistant Escherichia coli [28]. Recently, there was an increased interest in the use of a transperineal approach for prostate biopsy [28, 29]. Transperineal prostate biopsy has the advantage of avoiding penetration of rectal mucosa and thus minimizing inoculation of the prostate with bowel flora. Many published series of transperineal prostate biopsy reported their incidence of febrile complication with either zero or close to zero [28-30]. In this series, we use the prostate biopsy methodology of free-hand transperineal approach with general anesthesia, and no peri-procedure complication including infectious and anaesthetic complications was noted.

Several limitations of the present study needed to be mentioned. The study population consisted of patients referred to Chinese men in Eastern China, which could have induced selection bias. Second, patients with no lesion on mpMRI were excluded from the study, which could influence cancer detection rate of SB. Third, the sample size was small, which might have an effect on the results of the study.

\section{Conclusions}

This clinical study showed encouraging results for freehand transperineal targeted prostate biopsy guided with MRI-TRUS fusion imaging in Chinese men with previously negative biopsies and elevated PSA levels. MRI-TRUS fusion guided TB improves detection of clinical significant $\mathrm{PCa}$ in a repeat biopsy setting. Combination of TB and SB can maximize the PCa detection rate. PI-RADS score is the strongest predictor of $\mathrm{PCa}$ and significant cancer.

\section{Additional files}

Additional file 1: Table S1. Pathology results from systematic biopsy and targeted biopsy for prostate cancer. (DOCX $16 \mathrm{~kb}$ )

Additional file 2: Figure S1. Proportion of all cancers and clinically significant cancers stratified by PI-RADS score according to mpMRI scan. PCa, prostate cancer. (DOCX $138 \mathrm{~kb}$ )

\section{Abbreviations}

ESUR: European Society of Urogenital Radiology; mp: Multiparametric; MRI: Magnetic resonance imaging; PCa: Prostate cancer; PI-RADS: Prostate Imaging Reporting and Data System; PSA: Prostate-specific antigen; SB: Systematic biopsy; TB: Targeted biopsy; TRUS: Transrectal ultrasound

\section{Acknowledgements}

None.

Funding

No funding was obtained for this study.

Availability of data and materials

All data supporting our results can be found in confidential and deidentified databases located in the Department of Urology at Nanjing Drum Tower Hospital. Additionally, data supporting our findings is contained throughout our manuscript.

\section{Authors' contributions}

HL conception and design, acquisition of data, analysis and interpretation of data; drafting of manuscript. JZ conception and design, acquisition of data, or analysis and interpretation of data; drafting of manuscript. WW conception and design, acquisition of data, analysis and interpretation of data; drafting of manuscript. BZ acquisition of data, analysis and interpretation of data. JS acquisition of data, analysis and interpretation of data. DL analysis and interpretation of data. YF analysis and interpretation of data. XJ acquisition of data, analysis and interpretation of data. WZ acquisition of data, analysis and interpretation of data, supervision. HG conception and design, analysis and interpretation of data; drafting of manuscript, supervision. All authors have read and approved the final version of this manuscript. 


\section{Ethics approval and consent to participate}

The Nanjing Drum Tower Hospital Ethics Committee of Medicine approved this study. The approval number was 201,401,601. Written informed consent was obtained from all patients.

\section{Consent for publication}

Not applicable.

\section{Competing interests}

The authors declare that they have no competing interests.

\section{Publisher's Note}

Springer Nature remains neutral with regard to jurisdictional claims in published maps and institutional affiliations.

\section{Author details}

'Department of Urology, Drum Tower Hospital, Medical School of Nanjing University, 321 Zhongshan Road, Nanjing 210008, Jiangsu, People's Republic of China. ${ }^{2}$ Department of Radiology, Drum Tower Hospital, Medical School of Nanjing University, 321 Zhongshan Road, Nanjing 210008, Jiangsu, People's Republic of China. ${ }^{3}$ Department of Pathology, Drum Tower Hospital, Medical School of Nanjing University, 321 Zhongshan Road, Nanjing 210008, Jiangsu, People's Republic of China. ${ }^{4}$ Institute of Urology, Nanjing University, Nanjing 210008, Jiangsu, People's Republic of China. ${ }^{5}$ Department of Urology, the Affiliated Yixing people's Hospital of Jiangsu University, Yixing, Jiangsu 212000, China.

\section{Received: 4 August 2016 Accepted: 23 June 2017}

\section{Published online: 05 July 2017}

\section{References}

1. Pinsky PF, Black A, Parnes $\mathrm{HL}$, et al. Prostate cancer specific survival in the prostate, lung, colorectal, and ovarian (PLCO) cancer screening trial. Cancer Epidemiol. 2012;36:e401-6.

2. Taira AV, Merrick GS, Galbreath RW, et al. Performance of transperineal template-guided mapping biopsy in detecting prostate cancer in the initial and repeat biopsy setting. Prostate Cancer Prostatic Dis. 2010;13:71-7.

3. Puppo P. Repeated negative prostate biopsies with persistently elevated or rising PSA: a modern urologic dilemma. Eur Urol. 2007:52:639-41.

4. Walz J, Graefen M, Chun FK, et al. High incidence of prostate cancer detected by saturation biopsy after previous negative biopsy series. Eur Urol. 2006;50:498-505.

5. D'Amico AV, Tempany $C M$, Cormack $R$, et al. Transperineal magnetic resonance image guided prostate biopsy. J Urol. 2000;164:385-7.

6. Sartor $\mathrm{AO}$, Hricak $\mathrm{H}$, Wheeler $\mathrm{TM}$, et al. Evaluating localized prostate cancer and identifying candidates for focal therapy. Urology. 2008;72:512-24.

7. Rais-Bahrami S, Siddiqui MM, Turkbey B, et al. Utility of multiparametric magnetic resonance imaging suspicion levels for detecting prostate cancer. J Urol. 2013;190:1721-7.

8. Miyagawa T, Ishikawa S, Kimura T, et al. Real-time virtual Sonography for navigation during targeted prostate biopsy using magnetic resonance imaging data. Int J Urol. 2010;17:855-60.

9. Wysock JS, Rosenkrantz AB, Huang WC, et al. A prospective, blinded comparison of magnetic resonance (MR) imaging-ultrasound fusion and visual estimation in the performance of MR-targeted prostate biopsy: the PROFUS trial. Eur Urol. 2014;66:343-51.

10. Siddiqui MM, Rais-Bahrami S, Truong H, et al. Magnetic resonance imaging/ ultrasound-fusion biopsy significantly upgrades prostate cancer versus systematic 12-core transrectal ultrasound biopsy. Eur Urol. 2013;64:713-9.

11. Siddiqui MM, Rais-Bahrami S, Turkbey B, et al. Comparison of MR/ultrasound fusion-guided biopsy with ultrasound-guided biopsy for the diagnosis of prostate cancer. JAMA. 2015;313:390-7.

12. Zhang $\mathrm{Q}$, Wang $\mathrm{W}$, Yang $\mathrm{R}$, et al. Free-hand transperineal targeted prostate biopsy with real-time fusion imaging of multiparametric magnetic resonance imaging and transrectal ultrasound: single-center experience in China. Int Urol Nephrol. 2015:47:727-33.

13. Rosenkrantz AB, Kim S, Lim RP, et al. Prostate cancer localization using multiparametric MR imaging: comparison of prostate imaging Reporting and data system (PI-RADS) and Likert scales. Radiology. 2013;269:482-92.

14. Barentsz JO, Richenberg J, Clements $R$, et al. ESUR prostate MR quidelines 2012. Eur Radiol. 2012;22:746-57.
15. Weinreb JC, Barentsz JO, Choyke PL, et al. PI-RADS prostate imaging Reporting and data system: 2015, version 2. Eur Urol. 2016;69:16-40.

16. Harnden P, Naylor B, Shelley MD, Clements H, Coles B, Mason MD. The clinical management of patients with a small volume of prostatic cancer on biopsy: what are the risks of progression? A systematic review and metaanalysis. Cancer. 2008;112:971-81.

17. Ahmed HU, Hu Y, Carter T, et al. Characterizing clinically significant prostate cancer using template prostate mapping biopsy. J Urol. 2011;186:458-64.

18. Stamey TA, Freiha FS, McNeal JE, Redwine EA, Whittemore AS, Schmid HP. Localized prostate cancer. Relationship of tumor volume to clinical significance for treatment of prostate cancer. Cancer. 1993:71:933-8.

19. Dickinson L, Ahmed HU, Allen C, et al. Magnetic resonance imaging for the detection, localisation, and characterisation of prostate cancer: recommendations from a European consensus meeting. Eur Urol. 2011;59:477-94.

20. Wolters T, Roobol MJ, van Leeuwen PJ, et al. A critical analysis of the tumor volume threshold for clinically insignificant prostate cancer using a data set of a randomized screening trial. J Urol. 2011;185:121-5.

21. Radtke JP, Schwab C, Wolf MB, et al. Multiparametric Magnetic Resonance Imaging (MRI) and MRI-Transrectal Ultrasound Fusion Biopsy for Index Tumor Detection: Correlation with Radical Prostatectomy Specimen. Eur Urol. 2016;70(5):846-53.

22. Brock M, Loppenberg B, Roghmann F, et al. Impact of real-time elastography on magnetic resonance imaging/ultrasound fusion guided biopsy in patients with prior negative prostate biopsies. J Urol. 2015:193:1191-7.

23. Sonn GA, Chang E, Natarajan S, et al. Value of targeted prostate biopsy using magnetic resonance-ultrasound fusion in men with prior negative biopsy and elevated prostate-specific antigen. Eur Urol. 2014;65:809-15.

24. Borkowetz A, Platzek I, Toma M, et al. Comparison of systematic transrectal biopsy to transperineal magnetic resonance imaging/ultrasound-fusion biopsy for the diagnosis of prostate cancer. BJU Int. 2015;116:873-9.

25. Portalez D, Mozer P, Cornud F, et al. Validation of the European Society of Urogenital Radiology scoring system for prostate cancer diagnosis on multiparametric magnetic resonance imaging in a cohort of repeat biopsy patients. Eur Urol. 2012;62:986-96.

26. Sonn GA, Natarajan S, Margolis DJ, et al. Targeted biopsy in the detection of prostate cancer using an office based magnetic resonance ultrasound fusion device. J Urol. 2013:189:86-91.

27. Loeb S, van den Heuvel S, Zhu X, Bangma CH, Schroder FH, Roobol MJ. Infectious complications and hospital admissions after prostate biopsy in a European randomized trial. Eur Urol. 2012;61:1110-4.

28. Chang DT, Challacombe B, Lawrentschuk N. Transperineal biopsy of the prostate-is this the future? Nat Rev Urol. 2013;10:690-702.

29. Vyas $L$, Acher $P$, Kinsella J, et al. Indications, results and safety profile of transperineal sector biopsies (TPSB) of the prostate: a single centre experience of 634 cases. BJU Int. 2014;114:32-7.

30. Pepe P, Aragona F. Morbidity after transperineal prostate biopsy in 3000 patients undergoing 12 vs 18 vs more than 24 needle cores. Urology. 2013; 81:1142-6.

\section{Submit your next manuscript to BioMed Central} and we will help you at every step:

- We accept pre-submission inquiries

- Our selector tool helps you to find the most relevant journal

- We provide round the clock customer support

- Convenient online submission

- Thorough peer review

- Inclusion in PubMed and all major indexing services

- Maximum visibility for your research

Submit your manuscript at www.biomedcentral.com/submit
C) Biomed Central 